cemoti $\begin{aligned} & \text { Cahiers d'études sur la Méditerranée } \\ & \text { orientale et le monde turco-iranien }\end{aligned}$

25 | 1998

Les Ouïgours au vingtième siècle

\title{
L'arrière-plan historique du nationalisme ouïgour. Le Turkestan oriental des origines au XXe siècle*
}

\author{
Françoise AUBIN
}

\section{(2) OpenEdition}

Édition électronique

URL : http://journals.openedition.org/cemoti/42

DOI : $10.4000 /$ cemoti.42

ISSN : $1777-5396$

Éditeur

AFEMOTI

Édition imprimée

Date de publication : 1 janvier 1998

ISSN : 0764-9878

Référence électronique

Françoise AUBIN, «L'arrière-plan historique du nationalisme ouïgour. Le Turkestan oriental des origines au XXe siècle* ${ }^{*}$, Cahiers d'études sur la Méditerranée orientale et le monde turco-iranien [En ligne], 25 | 1998, mis en ligne le, consulté le 10 décembre 2020. URL : http://journals.openedition.org/ cemoti/42 ; DOI : https://doi.org/10.4000/cemoti.42

Ce document a été généré automatiquement le 10 décembre 2020.

Tous droits réservés 


\title{
L'arrière-plan historique du nationalisme ouigour. Le Turkestan oriental des origines au XXe siècle*
}

\author{
Françoise AUBIN
}

A la fin de notre millénaire, le pouvoir communiste chinois atteindra un demi-siècle d'existence. A peine cinq décennies, et déjà la force de persuasion du régime s'est faite assez pressante pour que ses principes fondateurs les plus théoriques soient reçus, à l'intérieur du pays et à l'étranger tout autant, comme des vérités irréfutables. L'exemple le plus frappant en est fourni par la théorie des nationalités, en chinois minzu (ou min-tsu), d'après un terme introduit depuis le Japon vers la fin du siècle dernier $^{1}$ : les citoyens de la République populaire de Chine (ou RPC) sont scindés en 56 groupes ethniques ou nationaux - c'est en effet ce même terme, minzu, qui désigne à la fois le peuple, l'ethnie, la nationalité (distinguée de la citoyenneté, à la différence de ce qui se fait en Occident). Plus de 90 pour cent de la population globale du pays est constituée par la nationalité-phare, support de toute modernité et de tout progrès; les Han ou Chinois de souche, amalgamés en une ethnie unique, du nord au sud, de l'est à l'ouest du pays, nonobstant des différences sociologiques, culturelles, linguistiques bien attestées. Par contre, les 55 autres nationalités sont considérées comme des "minorités ethniques". Réputées arriérées et dotées d'un folklore joyeux, voire licencieux, fortes les unes de plusieurs millions d'individus, les autres de quelques milliers seulement, elles occupent les immenses territoires colonisés à partir du XVIIIe siècle autour de la Chine propre. Aussi, lorsqu'on traite les Ouïgours, les Tibétains, les Mongols de "minorités ethniques", il faudrait se souvenir que ces-dites minorités étaient, il y a peu encore, majorité chez elles.

Le premier acte de foi du dogme ethnique de la RPC concernant le Xinjiang (ou Sinkiang, le Turkestan oriental) est que la minorité établie dans l'ensemble des oasis du Tarim, dans la moitié méridionale de la province, et dans les zones péri-urbaines du nord est celle des Ouïgours, une ethnie homogène, de religion musulmane (sunnite hanéfite), vouée à l'agriculture en terres irriguées. Le second point est que ces Ouïgours sont, à l'évidence de leur nom et de leur occupation du terrain, les descendants directs 
et légitimes des Ouigours du Moyen Age, ceux-ci se trouvant promus minorité ethnique d'un empire chinois éternel ${ }^{2}$. D'où découle le troisième point : les territoires soumis aux Ouigours médiévaux ont une vocation historique à rentrer dans la grande Chine.

D'ailleurs, et c'est là le quatrième point, les évidences archéologiques prouvent là une implantation bimillénaire du pouvoir chinois, donc une influence de sa civilisation continue depuis le début de notre ère, et, par voie de conséquence, une amitié tout aussi ancienne unissant autochtones et Han.

A contre-courant de telles vues, légitimées en Occident par le prestige entourant tout ce qui est chinois, la présente contribution a pour but de rappeler, premièrement, que le passé du Turkestan oriental n'est pas aussi simple que le veut la schématisation officielle de la RPC, et, deuxièmement, que la culture des Ouïgours modernes a une originalité propre, qui doit peu à celle de la Chine.

Les Ouïgours du passé ne sont pas les ancêtres des Ouïgours du présent

Le Turkestan oriental pré-gengiskhanide

Le Turkestan oriental est une de ces régions du monde dont le destin est si étroitement façonné par le cadre géographique qu'on ne peut comprendre le présent sans se référer aux contingences de son développement historique lié à son environnement. Il faut avoir sous les yeux une carte des reliefs de l'Eurasie pour saisir pleinement le problème du peuplement du Turkestan oriental - une région ingrate, à plateaux et cuvettes désertiques et semi-désertiques, bloquée par d'énormes chânes de montagnes parmi les plus hautes et difficiles du monde, qui s'étagent en vagues successives pour la mieux confiner, et, en son centre, des déserts terrifiants et gigantesques ${ }^{3}$. Or, paradoxalement ce pays, apparemment isolé et inaccessible, a été, au cours des siècles, une grande voie de passage des migrations ethniques et des courants de civilisation matérielle et intellectuelle que symbolise le terme de "Route de la Soie" (inventé à la fin du siècle dernier). Et ce fut aussi le cul-de-sac où les flots humains et les influences culturelles vinrent se superposer, se mêler, se fondre en de nouveaux modèles avant de mourir; ce fut aussi, depuis un siècle, un site privilégié d'explorations archéologiques, grâce auxquelles ont été exhumés des sables les restes de civilisations d'une richesse et d'une beauté à couper le souffle.

Etrangement, l'ancien peuplement indo-européen des oasis semblait, ces dernières années, en passe d'être oublié, en dépit du prestige de l'art gréco-bouddhique, par le public occidental et plus encore chinois, si l'on excepte, cela va de soi, les spécialistes du tokharien, de l'agnéen, du koutchéen, du sogdien et des peuples locuteurs de ces langues indo-européennes dans la partie orientale de l'Asie centrale. En est témoin l'étonnement suscité par les découvertes, faites dans la région de Hami entre 1978 et 1991 et popularisées au début des années quatre-vingt-dix, de momies qui, parfaitement conservées dans le sable, sont datables de 6000 à 2000 avant notre ère et qui présentent des caractéristiques indiscutablement europoïdes (long nez, dépressions oculaires profondes, pilosité blonde ou chataine). Et quasi-inquiète a été la surprise soulevée par la mise au jour, au printemps 1992 près de Turfan (chinois Turpan), de momies europoïdes plus tardives, des Ve-IVe siècle avant notre ère ${ }^{4}$.

La turcisation progressive, à partir du Ve siècle de notre ère, des peuples indoeuropéens qui étaient, à l'époque ancienne, les occupants sédentaires principaux de cette partie de l'Eurasie peut être d'autant mieux démontrée qu'elle s'est accompagnée d'une mutation linguistique totale ${ }^{5}$, ce qui ne sera pas le cas pour les Turcs médiévaux. Les vagues d'envahisseurs nomades non indo-européens, à dominante paléo-asiatique d'abord, puis proto-turque, turque, proto-mongole, sont identifiables grâce aux sources 
chinoises et à l'archéologie, à partir du IIIe siècle avant notre ère. De ce moment, les grand "empires des steppes" sont ceux des Xiongnu (ou Hsiung-nu, Hiong-nou), qui prolongent leur présence jusqu'au Ier siècle de notre ère ${ }^{6}$; des Ruanruan (ou Juan-juan) vers le Ve siècle ${ }^{7}$; des puissants Tujue (T'u-chüe, T'ou-kiue, ou Türküt) ${ }^{8}$, auteurs des plus anciens monuments écrits de langue turque au VIIIe siècle. Enfin la confédération des Ouïgours, qui, ayant anéanti dans les années 740 le second empire des Tujue, a laissé un souvenir assez prestigieux pour être réanimé au XXe siècle.

Les tribus ouiggoures ont pris leur origine sur le territoire de l'actuelle Mongolie, laquelle fut turque avant d'être mongole; et, unifiant là diverses ethnies sous leur domination, ils y ont maintenu un puissant empire des steppes, un khanat, qui a duré près d'un siècle (744/45-840), en relation d'alliance et de commerce avec la Chine des Tang et influencé profondément par elle, combinant nomadisme et vie urbaine, ayant adopté pour religion officielle le manichéisme? ${ }^{9}$. Leur pouvoir ayant été détruit par une vague d'envahisseurs kirghiz venus des rives du Iénisséï, les Ouïgours émigrèrent vers l'Ouest, et essaimèrent dans les oasis de la région la plus orientale du Turkestan et dans l'extrême ouest de la Chine. Désormais sédentarisés et voués à l'agriculture, pacifiques et tolérants, adhérant au bouddhisme ou au christianisme nestorien, sous l'influence des autochtones, à moins qu'ils ne soient restés fidèles à leur précédente foi manichéenne, ils ont laissé, autour de leur capitale Qocho, dans la région de Turfan et de Beshaliq (à 200 km à l'est de l'actuel Ürümchi), les restes d'une civilisation particulièrement brillante ${ }^{10}$.

La turquisation des oasis du Turkestan oriental est un processus irrémédiablement engagé, ainsi que la sédentarisation des nomades turcs. Quant à l'islamisation, si l'on peut en prendre pour point de départ la fameuse défaite des Chinois par les Arabes à Talas (Tarâz) en $751^{11}$, c'est au Xe siècle qu'elle se développe en Transoxiane (à l'ouest, donc, de l'actuel Xinjiang) sous les Samanides. En Kashgarie (dans le sud-ouest du Xinjiang) et dans la région de l'Ili et de l'Issyk-kul (à cheval sur la frontière septentrionale moderne), les acteurs de l'islamisation ont été, aux XI-XIIe siècles, une confédération à la teneur et à l'organisation fluctuantes, issue des Turcs Qarluq et marquée par la culture iranienne, les Qarakhanides (ou Karakhanides) ${ }^{12}$, qui, eux aussi, ont été les défenseurs d'une culture raffinée, dont témoignent deux des oeuvres les plus célèbres du passé littéraire turc en la région : le dictionnaire encyclopédique, le Dîwân lughât al-Turk de Mahmud al-Kâshgarî (ca. 1074) ${ }^{13}$ et la poésie didactique dépeignant la monarchie idéale, le Qutadku bilik de Yûsuf Khâss Hâjib ${ }^{14}$ de 1069. Mais la marche vers la conversion à l'islam n'est, alors, pas encore irréversible : le christianisme nestorien reprend occasionnellement, ainsi sous l'influence des nouveaux maîtres de la région à partir de 1130, les Qara-khitai, à dominante proto-mongole, chassés de la Chine dont ils avaient dominé le Nord (de 907 à 1125).

Le Turkestan oriental, des Gengikhanides à la disparition des Ouïgours A l'orée de l'époque gengiskhanide, vers le début du XIIIe siècle, la région est définitivement turquisée. Les Ouïgours sont restés une force culturelle, mais non plus politique comme auparavant, car ils sont soumis aux Qara-khitai. Tôt ralliés aux Mongols, avec lesquels ils entretenaient depuis longtemps des rapports commerciaux, et par choix volontaire, après avoir tué un gouverneur qara-khitai haï, en 1209, soit trois ans après le quriltai au cours duquel Temüjin avait pris le nom de Gengis-khan et fondé l'Etat mongol, ils se trouvent être les premiers membres d'une culture urbaine vassaux des Mongols. Ils vont être, pour ceux-ci, des initiateurs de culture, en concurrence et parfois en compétition avec les chefs chinois des groupes d'auto- 
défense de la Chine du Nord, eux aussi gagnés au parti du Grand khan. Ainsi, ils contribuent à l'introduction et au maintien d'un système administratif, fiscal et commercial dans le jeune empire gengiskhanide ${ }^{15}$ et, surtout, ils sont les promoteurs de l'écriture traditionnelle mongole, en usage jusqu'à nos jours en Mongolie intérieure ou à nouveau de nos jours en République de Mongolie, adoptée aussi et adaptée par les Mandchous au XVIIe siècle. Cette écriture ouïgoure, avant de devenir écriture mongole au XIIIe siècle et de jouer dès lors un rôle décisif dans l'histoire culturelle des steppes, a connu elle-même une curieuse évolution : dérivée de l'alphabet araméen, par le relais sogdien, elle était primitivement notée, à la manière sémite, horizontalement et de la droite vers la gauche, comme l'arabe qui en est dérivé aussi. Sous l'influence chinoise semble-t-il, à l'époque du royaume de Qocho avant l'occupation mongole, elle bascule de $90^{\circ}$ : elle s'écrit donc dorénavant à la verticale, mais conservant l'orientation qu'elle avait à l'horizontale, elle se lit en commençant par la gauche de la feuille ${ }^{16}$ (alors que le chinois se lit anciennement à la verticale certes, mais de la droite vers la gauche). A ce propos, il est amusant de constater l'usage négatif qui est fait du terme "Ouïgour" dans l'affirmation identitaire de la République mongole post-communiste : les partisans du rétablissement de cette écriture traditionnelle, vermiculaire et verticale, comme écriture officielle l'appellent "écriture nationale", alors que ses adversaires, ceux qui souhaitent le maintien de l'alphabet cyrillique, dénomment l'écriture traditionnelle "écriture ouïgoure" afin de montrer par ce nom qu'elle est d'origine tout aussi étrangère que le cyrillique.

Une autre subversion de la tradition, jusqu'à maintenant impossible à dater avec précision ni à expliquer, est celle qui a frappé l'appellation des Ouïgours en chinois : au XIIIe siècle, sous la dynastie mongole des Yuan, le nom qui désignait les Ouïgours dans les textes chinois, Hui-he, en vient à désigner les musulmans d'Asie centrale et du Moyen-Orient en général, ce qui prouve l'islamisation d'une partie des Ouïgours assez notable pour être remarquée dans l'Empire du Milieu. Dans les siècles suivants, le terme, sous la forme Hui-hui, va s'appliquer à l'umma dans son ensemble; et son dernier avatar est, à l'époque communiste, sous la forme abrégée Hui, la désignation de la "nationalité" (minzu) des musulmans de langue chinoise. Cependant que les Ouïgours encore bouddhistes au XIIIe siècle sont nommés Weiwu, et plus tard Weiwuer, le nom chinois des actuels occupants des oasis du Xinjiang.

$\mathrm{Au}$ XIIIe siècle, la région ouïgoure de Turfan rentre théoriquement dans le khanat des Chagataides (lequel dure jusqu'à sa prise par Tamerlan en 1370), fondé par Chagatai, le troisième fils de Gengis-khan. Mais jusqu'aux premières décennies du XIVe siècle, l'influence de la Chine et de sa dynastie mongole des Yuan (issue du quatrième fils de Gengis-khan) reste là prépondérante ${ }^{17}$. Cependant un islam toujours fortement marqué par le soufisme gagne un terrain cette fois-ci définitif, d'abord en Transoxiane, la partie la plus occidentale du khanat Chagataide, puis dans le Moghûlistan (région de l'Issykkul, du Semirechie, de Kashgar), et partout le nomadisme régresse sous l'influence de la nouvelle religion et le respect de la sharî'a remplace l'observance du droit coutumier post-mongol, le töre. En Ouïgouristan (région de Turfan et de Hami), le bouddhisme reste un rival solide jusqu'au début du XVIe siècle. Mais vers le milieu du siècle, la mention d'une ethnie spécifique, caractérisée par le bouddhisme, a disparu ${ }^{18}:$ les Ouïgours ont été absorbés dans une nouvelle société d'origine cosmopolite, comme les Sogdiens l'ont été avant eux et tous les autres peuples nomades et sédentaires qui sont apparus au cours des siècles dans le bassin du Tarim (et dont quelques-uns seulement ont été mentionnés ci-dessus). 
Les réels descendants directs des Ouïgours médiévaux ne sont plus, à partir du XVIe siècle au moins, les habitants des oasis dudit Ouïgouristan, mais, semble-t-il, un minuscule noyau de quelques milliers d'individus logés aux marges de la Chine, dans l'actuelle province du Gansu, les Sarigh Yugur (dénommés aussi Yugur noirs ou Yugur occidentaux), de langue turque et de religion bouddhique ${ }^{19}$.

Le Turkestan Oriental, de la domination oïrate à la domination chinoise

Ce n'est cependant pas encore là la fin de l'aventure. Au XVIIe siècle, une nouvelle vague d'invasion s'abat sur le Turkestan oriental : celle des Mongols occidentaux, des adhérents fervents du bouddhisme tibétain (ou lamaïsme) depuis les années 1620, porteurs d'une civilisation mongole riche et originale, qui sont connus de l'histoire sous des noms variés, propres à embrouiller le récit - Oïrat de leur nom générique; Choros du nom de la tribu de leur maison royale; Jûngar du nom de leur khanat (nous disons Djoungar ou Dzoungar);ou encore Torgût ou Ölööt du nom de leurs sous-tribus, et de ce dernier nom, les observateurs européens de l'époque ont fait Eleuthes. A partir du XVe siècle, ils occupent la région au nord des Tianshan (la chaîne qui coupe en deux l'actuel Xinjiang) - région qui, dans l'historiographie occidentale, prendra le nom de "Dzoungarie" (du nom des Jûngar). Et, de là, ils opèrent, sous la pression des événements extérieurs, de ces vastes glissements de population habituels dans l'histoire des steppes : ainsi en 1634, jusqu'au Köke-nûr (Koko-nor) et au Tibet; en 1678, jusqu'en Kashgarie, où ils sont attirés par des dissensions entre dirigeants religieux. Car, en cette partie du monde turkestanais, la politique s'est structurée, depuis le XVIe siècle, autour de lignées de khôja (ou khwâja) naqshbandis, des sayyid qui se glorifient de descendre directement de Mahomet par l'imâm Husain, le deuxième fils de Fatimâ. Le fondateur de ces lignées est un Samarcandais, Makhdûm A`zam (mort en 1542), un thaumaturge influent vers la fin de sa vie au Turkestan oriental, auprès du dernier souverain chagataide effectif siégeant à Yarkand. Le charisme du khôja est, à la mort de celui-ci, revendiqué par deux de ses fils (nés de mères différentes) qui fondent des souslignées agressivement rivales, de Samarcande au Turkestan oriental, Ishâq et Ishân. Ceux-ci, outre qu'ils sont leaders religieux, se conduisent en leaders politiques à la suite de l'évanouissement quasi total du pouvoir chagataide ${ }^{20}$.

C'est après un appel du petit-fils d'Ishân, Âfâq, au Dalaï-Lama que les Oïrats (ou Jûngar) foncent sur Kashgar en 1678 et intronisent, en 1679, Âfâq souverain. Pour les autochtones, la domination oïrate a-t-elle été oppressive ou, du moins, coercitive ? Il est délicat d'en juger d'une façon générale. Envisagée du point de vue mongol, la région est soumise à une suprématie suzeraine assez stricte, à laquelle les musulmans doivent se soumettre, mal gré plutôt que bon gré. Et l'on dit que le nouveau pouvoir monte une garde si étroite aux voies de passage vers l'ouest que les pèlerinages des musulmans de Chine propre (les ancêtres des actuels "Hui") aux centres spirituels de leur foi, Boukhara et Samarcande, en deviennent difficiles. Mais du point de vue musulman, ces kâfir haïs sont de très lointains suzerains et tout se passe comme s'ils n'existaient pas. La lutte sanglante entre les deux clans de khôja, celui de Qarataghliq, "la Montagne noire" issu de Ishâq, et celui de Aqtaghliq, "la Montagne blanche" remontant à Ishân, l'un basé à Kashgar, l'autre à Yarkand, et les raids des nomades kirghiz (ou kazakh?) sont, pour les habitants des oasis, des réalités plus préoccupantes. Cela jusqu'à ce que les Ö̈rats réapparaissent en 1713, pour imposer de nouveau leur pouvoir et un tribut plus lourd. Dorénavant les élites turkestanaises doivent rendre visite périodiquement à leur suzerain, au Nord, dans la vallée de l'Ili, et laisser des otages auprès de lui. 
En 1754, l'arrivée des armées impériales chinoises - ou, plus exactement, sinomandchoues, puisque la dynastie régnant à Pékin, celle des Qing (Ch'ing ou Ts'ing, 1644-1911), est d'origine mandchoue - met fin à une situation politique particulièrement instable. Les Oïrats sont physiquement exterminés, à partir de 1754, par les armées impériales et par une épidémie meurtrière; et le Turkestan oriental est annexé à l'Empire chinois en $1759^{21}$. En pratique, la suzeraineté sino-mandchoue s'exerce sur la moitié Sud du pays, comme à l'époque de la domination oïrate, sous la forme d'un prélèvement fiscal; et les beg, fonctionnaires locaux autochtones, continuent, comme auparavant, à faire marcher l'administration dans les villes et les oasis qui en dépendent. Dans le nord du pays, en Dzoungarie, des colonies civiles et militaires assurent à la fois la sécurité et la mise en valeur de la région ${ }^{22}$.

Les forces ethno-politiques de l'époque contemporaine commencent à s'organiser, mais elles ne seront institutionnalisées que dans le dernier quart du XIXe siècle, lorsque la Chine aura reconquis, en 1876-77, la région qui lui avait totalement échappé depuis plus d'une décennie. En effet, la lignée de Makhdûmzâda, les khôja de Kashgar et de Yarkand, et leurs partisans, pour beaucoup des marchands andijanais réfugiés dans le khanat de Kokand (la ville-capitale est à 450 km environ à vol d'oiseau à l'ouest de Kashgar, à quelque $110 \mathrm{~km}$ au sud-ouest d'Andijan, dans la vallée du Ferghana), avaient conduit de là d'incessants jihâd (guerres saintes) contre l'occupant chinois, le contraignant à une dépense coûteuse et malaisée : Pékin est, ne l'oublions pas, distant de Kashgar de quelque cinq mois de marche à pied. Les soulèvements anti-chinois, animés par les forces des khôja, s'étaient succédé dans la première moitié du XIXe siècle : en 1815, 1820 à 1828, 1847, 1855, 1862 ${ }^{23}$. Enfin, profitant de l'affaiblissement du pouvoir sino-mandchou, empêtré dans les soulèvements de la Chine propre (Taiping 1751-65, Nian 1853-68, musulmans du Yunnan 1855-72, musulmans du Nord-Ouest de la Chine 1862-72), une sorte d'aventurier de Kokand, Ya'kûb-beg (1820-1877), s'impose comme souverain d'une Kashgarie indépendante, de 1866 à 1876. Autoproclamé amîr alMu'minîn, "Commandeur des Croyants"24, et atalik ghâzi $i^{25}$, prenant la sharî̀ a comme règle de droit, il paraît être le chef d'un Etat musulman assez respectable pour que la Turquie lui promette son soutien. Et la renaissance matérielle, intellectuelle, religieuse qu'il semble imprimer à son royaume donne aux Britanniques une telle illusion de stabilité qu'ils commencent à s'engager à ses côtés, alors que la chute est déjà proche. Le système d'espionnage généralisé qu'il a mis en place, et qui restera un trait caractéristique du Xinjiang jusque dans le XXe siècle, ne suffit pas pour prévenir le mécontentement populaire, attisé par les guerres incessantes, les impôts oppressifs, la débâcle économique. Les Russes viennent d'atteindre l'Asie centrale et menacent la Kashgarie sur son flanc occidental. De l'autre côté, les Sino-Mandchous, vainqueurs de toutes les révoltes internes, reprennent, dans une avance implacable, les terres désormais nécessaires à la protection de leurs zones frontalières les plus éloignées ${ }^{26}$. En 1884, le Turkestan oriental est incorporé dans l'Empire chinois, comme province du Xinjiang (ou Sinkiang), littéralement "Nouvelles marches frontalières", avec une capitale au nord des monts Tianshan, à Ürümchi ("Ouroumchi", comme on l'écrit dans nos media, ou Urumqi comme l'écrivent les Chinois de RPC). Les limites de la zone d'influence chinoise s'étant heurtées à celles des empires russe et britannique, elles se sont matérialisées en des frontières, plus ou moins précises en ces lieux faiblement habités; dans leur ensemble, elles suivent les lignes de crêtes naturelles, mais elles coupent aussi, surtout au nord-ouest, à travers des voies séculaires de transit, sectionnant des territoires coutumiers de nomadisation des Kazakhs, ce qui fournira 
matière à des frictions de voisinage. Le Xinjiang est devenu depuis les dernières décennies du XIXe siècle, et seulement alors, une entité géo-politique.

La réalité sociologique

Et quelle appellation générique?

Dans les sources chinoises antérieures au XIXe siècle, le Turkestan Oriental est tout simplement désigné comme "la Contrée d'Occident" Xiyu (Hsi-yü). Mais jusqu'à l'instauration du Xinjiang en 1884, aucune unité n'a jamais existé dans l'ensemble de la région, non plus qu'aucun nom ethnique ou géographique global. Dans l'érudition occidentale, l'on distingue, à l'aide de termes arbitraires, de grandes sous-régions ${ }^{27}$. A l'est, c'est, en souvenir des Ouïgours médiévaux qui ont régné là, l'Ouïgouristan, qui, autour des villes de Turfan et Qomul (Hami en chinois), a toujours entretenu des rapports commerciaux et culturels étroits avec la Chine voisine. Au nord de la chaîne des Tianshan, qui scinde la province en deux, la Dzoungarie est un ancien domaine de nomades. Au sud de la chaîne, le bassin de Tarim formait la Kashgarie, dont le sud, avec la ville de Khotan, était jadis tourné plutôt vers l'Inde, alors que le nord et l'ouest étaient dans la continuité culturelle et spirituelle du Turkestan occidental. Le centre en est un immense plateau désertique, le Takla-makan, cerné d'un chapelet d'oasis sur les eaux résurgentes au piémont des hautes chaînes bordières. Il est bien connu que, jusque très avant dans notre siècle, les autochtones se désignaient par le nom de la ville capitale de leur oasis : Kâshgarlik "ceux de Kashgar", Yârkandlik "ceux de Yarkand", etc. Au XIXe siècle, des noms génériques ont été trouvés pour désigner sur place la Kashgarie, mais ce n'était qu'une addition de villes : généralement les "Six cités" ou "Hexapole" Altashahr (variante turkestanaise de Altishahr) ${ }^{28}$, ou les "Sept cités" Yedishahr (ou Yitishahr) à l'époque de Ya'kûb-beg. Pour ceux d'entre les Kashgariens qui, après la destruction du khanat oïrat, avaient émigré en Dzoungarie et y avaient fait souche comme agriculteurs ou comme commerçants, le nom générique est celui de Taranchi "agriculteur" (mais le nom de Sartes, que l'on trouve dans la littérature occidentale sur le sujet pour désigner les Turkestanais d'origine russe installés au Xinjiang n'a pas été reçu par les autochtones) ${ }^{29}$.

S'il leur fallait marquer leur appartenance commune, les Turkestanais, prenant l'Islam comme signe identitaire, se disaient Musulman khalk. Quant à la dénomination géographique de Turkistân, dont nous avons fait "Turkestan", elle vient du persan : "Pays des Turcs", et elle a été reprise au XIXe siècle par les intellectuels turkestanais. De nos jours encore, les émigrés ne nommeront leur pays d'origine que "Turkestan oriental", refusant l'appellation du Xinjiang comme étant celle qu'a imposée le colonisateur et qui est chargée d'une connotation avilissante. L'on remarquera que bien plus dépréciatif est le terme dont les Chinois de l'époque impériale avaient l'habitude de désigner les musulmans du Xinjiang : chantou (ch'an-t'ou), "les têtes enturbannées". Cependant, le nationalisme turkestanais était en gestation au début de l'époque républicaine (à partir de la fin de 1911) ${ }^{30}$ et, sous l'influence des théories occidentales de la nationalité, le besoin se faisait sentir de trouver un nom générique ayant la valeur d'une devise de rassemblement identitaire. Le choix du nom prestigieux de "Ouïgour" a été un coup de génie, mais il est venu de l'extérieur. Les conditions de son lancement, restées longtemps hypothétiques, paraissent maintenant établies : cet ethnonyme a été retenu lors d'un des premiers congrès des nationalités de la toute jeune Union Soviétique, celui qui s'est déroulé en 1921 à Tashkent, le haut-lieu de l'éducation et de la propagande communiste en Asie centrale. Le nom était alors destiné à constituer en une "minorité ethnique" spécifique les émigrés venus des oasis du Xinjiang dans l'Asie 
centrale russe ${ }^{31}$. La suggestion en avait été faite par le linguiste russe Sergej (prononcer Sergueï) Efimovich Malov (1880-1957). Si la Grande Encyclopédie Soviétique prétend que Malov a pris cette initiative en $1921^{32}$, c'est tout bonnement pour la faire coïncider avec le congrès de Tashkent. En fait, Malov avait déjà employé l'ethnonyme "Ouïgour" pour désigner la langue du Sud-Xinjiang dans les rapports de ses deux premières expéditions, publiées dès 1912 et 1914.

D'ailleurs, en remontant quarante ans plus haut dans le passé, on découvre une autre mention du terme Ouigour, appliqué à la langue - comme va le faire Malov - dans le rapport de l'expédition menée au coeur de la Kashgarie de Ya'kub-beg en 1873, sous la conduite de Sir Forsyth : "The language of the people of Kashgaria (= le Sud du Turkestan Oriental) is the Uighur dialect of Turki"; et cette langue est mise par l'auteur en équation avec celle des Ouïgours médiévaux ${ }^{33}$. L'idée d'une langue "ouïgoure" commune aux habitants des oasis du Turkestan oriental (héritière ou non du ouïgour médiéval) traînait donc dans les écrits occidentaux de la fin du XIXe et du début du XXe siècle, de même que l'expression "Route de la Soie", forgée dans l'Europe de la Belle Epoque : et l'on sait le succès qu'ont remporté l'un et l'autre terme en Extrême-Orient et en Asie centrale au cours du XXe siècle.

Ce sont les Russes qui ont répandu l'ethnonyme au Xinjiang lorsque leur influence s'y est développée à la fin des années vingt et au début des années trente. Et il est diffusé à Ürümchi en 1934 par une Association pour la promotion de la culture ouïgoure, puis, bientôt, officialisé dans un communiqué cosigné par le nouveau gouverneur chinois, Sheng Shicai (Sheng Shih-ts'ai, lequel a pris le pouvoir en avril 1933) et le vicegouverneur, un natif de Qomul (ou Hami), Khojâ-Niaz, après que, notons-le, Garegin Apresov a été nommé consul-général soviétique à Ürümchi (printemps 1933) et devient, dès lors, la véritable éminence grise du pays (il tombera lui-même victime des purges staliniennes en 1937) ${ }^{34}$. Le terme sert dorénavant d'assise à une prise de conscience communautaire de gens accoutumés, jusqu'alors, à ne s'identifier qu'à une oasis. Il est, de plus, enrichi par la théorie des nationalités qui se répand à ce moment, venant de divers bords, soviétiques notamment, et qui rencontre grand succès parmi les hommes politiques.

La médaille a cependant son revers : l'identification entraîne le sectarisme. L'exclusion des différences et d'autrui se trouve justifiée, et les virtualités d'union panislamique neutralisées par avance. Pour la plupart des nationalistes turkestanais qui s'expriment après 1934, l'autonomie réclamée aux Chinois doit, cela va de soi, donner aux Ouïgours la prééminence sur les autres peuples musulmans du Xinjiang.

Depuis que le gouvernement populaire a pris comme l'un des articles de son credo l'étiquetage ethnique, déjà préparé au Xinjiang par le gouverneur des années trente Sheng Shicai, nul ne songe plus à mettre en doute le mythe de la filiation directe entre Ouigours du IXe siècle et autochtones du Tarim, dénommés pareillement en chinois moderne Weiwuer ${ }^{35}$. La gloire des khanats ouïgours de l'époque médiévale se trouve donc confisquée au profit de la RPC, qui proclame les anciens peuples nomades, et, par voie de conséquence, les territoires qu'ils ont couverts, partie intégrante de la nation chinoise, et les restes archéologiques d'objets chinois preuves d'une présence chinoise bien établie en Asie centrale depuis le premier siècle avant notre ère, des arguments qui ont été, des années soixante aux années quatre-vingt, mis et remis sans trêve en avant dans les contestations territoriales avec la défunte URSS ${ }^{36}$. Dans la ligne de revalorisation des cultures indigènes qui a suivi les persécutions de la Révolution 
culturelle, ne voit-on pas le dictionnaire d'al-Kâshgari publié en traduction ouïgoure moderne et en chinois, à titre $d^{\prime}$ "ouvrage célèbre de la nationalité ouigoure"? ${ }^{37}$ Et pourtant al-Kâshgari était un noble qarakhanide et non pas un ouïgour. Le royaume ouïgour ne s'est d'ailleurs pas étendu jusqu'à la Kashgarie. Beaucoup d'autres peuples, indo-européens, turcs et mongols, ont contribué à façonner les ethnies turkestanaises actuelles, lesquelles ne sont pas aussi homogènes que le nom ethnique commun de "Ouïgour" voudrait le faire croire, ni culturellement et linguistiquement, ni ethniquement ${ }^{38}$, ni idéologiquement et politiquement, car la vieille différenciation entre Ouïgouristan et Kashgarie s'est maintenue jusqu'à nos jours. Les linguistes occidentaux, qui, jadis, dénommaient "türki oriental" la langue d'intercommunication du Turkestan oriental, parlent maintenant de "nouvel ouigour"39.

La culture traditionnelle des oasis turkestanaises

Dans la bataille idéologique qui oppose, de nos jours, les intellectuels nationalistes ouïgours au gouvernement chinois, les premiers prétendent être les occupants originels du territoire, et le second répond que son empire s'est étendu sur la région bien avant les Ouïgours, depuis le siècle précédant le début de notre ère. L'un et l'autre argument sont contournés. Nous l'avons vu : si l'on remonte dans le passé plus haut que les khanats turcs, les autochtones que l'on trouve installés dans les oasis sont des IndoEuropéens ${ }^{40}$. Quant à la présence de l'Empire chinois durant ses périodes d'expansionnisme glorieux, sous les Han et sous les Tang, elle n'a été qu'intermittente, fortement militaire et entrecoupée par les siècles où la Chine même était, dans sa partie septentrionale ou en totalité, soumise à des dynasties turques ou mongoles. Et il ne faut pas oublier, non plus, que les Tibétains aussi ont occupé le pays à la fin du VIIe siècle et un siècle entier entre le milieu du VIIIe et le milieu du IXe siècle ${ }^{41}$.

Alors que la culture des Ouïgours médiévaux a été notoirement influencée par la Chine des Tang, celle des Ouïgours modernes ne relève que du monde centre-asiatique. Cette culture traditionnelle des oasis turkestanaises nous est connue beaucoup mieux qu'on ne pourrait l'attendre d'un pays aussi excentré et isolé. Car la richesse de son passé archéologique, sa vie en marge du monde moderne, le défi sportif que représente la traversée des chaînes de montagnes et des déserts qui la protègent, l'appel missionnaire aussi, tout s'est ligué pour attirer voyageurs, explorateurs, érudits de terrain au XIXe et au XXe siècles ${ }^{42}$.

Un document de choix, souvent ignoré par les auteurs anglo-saxons, est fourni par l'Etude ethnographique et sociologique de Fernand Grenard (Grenard, 1898), membre de la Mission scientifique dans la Haute-Asie commandée par Jules-L. Dutreuil de Rhins entre 1890 et 1895 (le malheureux Dutreuil de Rhins terminant sa vie, assassiné aux marges du Tibet, en 1893). Grenard présente, après une brève vue de la géographie et de l'histoire du pays, le caractère des autochtones, la culture matérielle - habitat, costume, nourriture -, les relations familiales, la vie sociale, l'agriculture, l'artisanat et l'industrie naissante, le commerce, la religion et le "clergé", les rites du mariage, l'administration impériale en cette fin de dynastie Qing : au total plus de 300 pages d'une documentation irréprochable. Des récits ou rapports plus anciens, tels ceux de Robert Shaw (1871/1984) et de T. Douglas Forsyth (1875) datant de l'époque de Ya`kûbbeg, de Aleksei N. Kuropatkin (1882, voyage de 1876), ou plus tardif, comme ceux du lieutenant P.T. Etherton (1911, témoignage de 1909) et de Albert von Le Coq (1928, expédition archéologique de 1905-1906) ajoutent des touches par-ci, par-là. K.B. Warikoo (1985) en fait une synthèse commode (mais il ignore Grenard); et l'historien 
japonais Saguchi Tôru (1978) étudie habilement la vie politique du Xinjiang sous domination Qing, en résumé de ses nombreuses publications en japonais.

Pour l'époque de la République, une étude, marquante par la clarté de ses détails, sur l'agriculture des oasis est due à un missionnaire catholique allemand, ayant oeuvré au Xinjiang de 1922 à 1939, comme membre de la Société du Verbe Divin (SVD ou Steyl), une congrégation allemande qui, en 1922, avait reçu le champ de mission turkestanais des mains de la Congrégation belge de Scheut (ou CICM), laquelle en avait précédemment la charge : le P. Golomb (1959), qui a opéré à partir d'Ürümchi. Les missions protestantes, suédoises d'origine, ont été, elles, actives à Kashgar; et c'est auprès d'elles que celui qui allait devenir un turcologue et un homme politique éminent, le Suédois Gunnar Jarring, s'est initié à la langue et aux coutumes locales. On lui doit une abondante collection de petites monographies ethnologiques, sur des sujets parfois inattendus, appuyées de textes originaux en türki (par exemple Jarring, 1979-80 \& 1991-92). Un ethnologue roumain a exposé la vie de l'oasis de Turfan vers les débuts du régime populaire : Tancred Banateanu (1960-61). Parmi les voyageurs qui ont eu d'autres soucis que trajets, étapes et chasse, il faut citer les demoiselles Cable et French (1942/1984), des missionnaires évangéliques à l'esprit indépendant et frondeur, qui, travaillant sous l'égide lointaine de la China Inland Mission, ont posé un regard chaleureux sur les populations locales qu'elles ont côtoyées lors de leurs campagnes d'évangélisation au Xinjiang en 1926 et, de nouveau, en 1935. Plus tard, l'ethnologie soviétique, active au Xinjiang avant la rupture sino-soviétique, a privilégié, à son habitude, la culture matérielle : Mmes Zaxarova $(1959,1963,1965)$ et Chvyr (1986 et un livre en 1990) se sont fait une spécialité du vêtement, de la coiffure et de la parure au Turkestan oriental et de leur comparatisme à travers l'Asie centrale.

La synthèse générale est à rechercher chez Thomas Hoppe (1995) - malheureusement son système de transcription du ouïgour, transposé du système utilisé en RPC pour noter les langues autochtones non chinoises, risque de décourager le lecteur, ainsi xeyh là où nous écrivons shaykh. Enfin, une talentueuse ethnologue d'origine hongroise, Mme Ildikó Bellér-Hann, capable de s'exprimer en ouïgour (ce qui n'est pas fréquent), enquête sur la vie dans les villages du Xinjiang méridional dans les années quatrevingt-dix ${ }^{43}$.

L'islam dans la vie turkestanaise

En matière d'ethnologie turkestanaise, la grande nouveauté de ces deux dernières décennies a été la découverte du rôle profond du soufisme, grâce à cinq turcologues armés d'une érudition sans faille : un Japonais qui s'appuie sur des textes türki du siècle dernier, Hamada Masami (1978); un Suédois, dont il a déjà été ici fait mention, Gunnar Jarring (1985-86 : de lui, une traduction de deux petits textes traitant des dervish et des qalandar); un savant américain, trop tôt disparu en 1984, qui savait manier toutes les sources en langues originales d'une extrémité à l'autre de l'Asie, Joseph Fletcher (1975, 1986, 1994, 1995); un Coréen spécialiste entre autres du Turkestan oriental à l'époque des khôja, Kim Ho-dong (1993); un jeune turcologue français qui suit la trace des confréries soufies de la Turquie à l'Inde, en passant par l'Asie centrale, Thierry Zarcone (1996).

Car l'on en était resté jusqu'alors à une description extérieure de la religion, remontant aux observations de la mission française des années 1890 et de quelques remarques de voyageurs, incidentes et souvent contradictoires. Pour tous, le trait le plus visible de l'islam turkestanais était son amorphisme, ainsi que l'atonie générale de la vie culturelle et spirituelle. Avant l'annexion chinoise, les mots d'ordre se prenaient à 
Boukhara et Samarcande; puis les autorités d'occupation s'étaient appliquées, remarque Grenard, à tuer toute initiative. Tendance bien en accord avec la douceur des moeurs relevées par les observateurs, l'insouciance, le refus de l'effort.

L'alphabétisation était d'ailleurs des plus restreintes, et l'instruction, même primaire, peu répandue. Il y avait pourtant, en théorie, une école, maktab, rattachée à chaque mosquée, c'est-à-dire un mollâ prêt à enseigner une mémorisation mécanique du Coran, et une mosquée à chaque coin de rue. Les madrasa paraissent avoir été davantage des hôtelleries que des collèges théologiques, et dans les meilleurs des cas, à Yarkand par exemple, les étudiants apprenaient par coeur le Coran, le droit coranique et lisaient quelques ouvrages persans faciles. Car le persan était plus ou moins bien parlé dans les milieux cultivés.

Les intellectuels qui ont pris part aux rébellions des années trente de notre siècle ${ }^{44}$ avaient rapporté leurs idées nouvelles de leurs études dans les centres musulmans étrangers, de Turquie, d'Inde, d'Afghanistan, ou plus souvent des centres islamiques de l'Union Soviétique. Pour le menu peuple, comme en toute région isolée du monde musulman, l'ouverture sur l'extérieur passait par l'expérience du hâjj, le pèlerin retour de La Mecque, qui avait réussi un périlleux voyage à travers les massifs du Kunlun et du Karakoram, pour gagner, à travers l'Inde, la voie maritime vers Djeddâ.

Les essais d'écoles chinoises n'ont eu aucun succès jusqu'au gouvernement de Sheng Shicai, entre 1933 et 1944, non plus que la pratique du chinois. L'un des reproches que les leaders insurgés font au Guomintang est de ne donner aux autochtones aucune chance de promotion dans la structure administrative. L'ignorance de la vie publique était telle qu'en 1933, à Kashgar - la ville où la mission protestante suédoise avait introduit pourtant des effluves de culture occidentale - le peuple se croyait encore gouverné par la dynastie Qing, tombée pourtant 22 ans plus tôt! $4^{45}$.

L'indifférence à l'égard de l'éducation est à imputer, en grande partie, à l'extrême fragilité de la cellule familiale et du lien matrimonial. La facilité et la fréquence des divorces, en contraste avec leur rareté chez les nomades kazakh du nord de la province et les Dounganes (Chinois musulmans installés au Xinjiang), étaient des sujets d'étonnement pour les Occidentaux et pour les Musulmans étrangers. La polygamie et les mariages temporaires étaient fréquents chez les commerçants (et tout Ouïgour était plus ou moins marchand), mais sous la forme de foyers multiples éparpillés en diverses oasis ou quartiers d'une ville. L'enfant devait apprendre tout jeune à ne compter que sur lui-même, perdant vite de vue ses parents et surtout ses frères et soeurs, car la majorité nuptiale était à 12 ans pour les garçons, 10 ans pour les filles. L'individualisme dans la vie familiale faisait écho au particularisme des villes dans la vie nationale. Si la femme n'avait pas de biens propres, elle se trouvait dans la misère à l'abandon de son mari (en principe, les enfants se partageaient entre les époux, les garçons au père, les filles à la mère, celle-ci ne recevant une pension que si, par accord particulier, elle élevait les fils). Mais, en revanche, elle jouissait d'une liberté personnelle et patrimoniale inimaginable en pays musulman : possibilité d'obtenir le divorce bien audelà de l'autorisation coranique, tolérance des moeurs, disposition de soi et de ses biens après la dissolution du mariage, droit au remariage (les femmes veuves ou divorcées ayant procréé étant plus prisées que les jeunes filles), droit de participer à la vie sociale en ayant le pas sur le mari, de contrôler les opérations domestiques, de tenir commerce, de venir à la succession du mari, même en présence d'enfants ( $1 / 8$ des biens dans ce cas, sinon 1/4) et à la succession du père en l'absence de frères. L'obligation du 
voile est diversement évaluée par les témoins, sans doute en dépendance des lieux et des temps, mais en général c'est une mousseline assez légère, souvent relevée.

La seule contrainte coranique qui pesait sur la validité du mariage de la femme était, mis à part l'empêchement de proximité parentale au 3e degré jouant pour les deux sexes - la prohibition de l'union avec un infidèle, qu'il soit chinois, tibétain, hindou ou mongol; mais cette interdiction pouvait être tournée par la faveur d'un mollâ complaisant. La prostitution était considérée comme une spécialité des Chinoises; quand une Musulmane y cédait, les mollâ réclamaient sa mort; mais pour les gens du peuple, il n'y avait aucune répugnance à l'épouser, le mariage effaçant les fautes passées.

Le degré d'adhésion au culte a été apprécié diversement selon les auteurs et selon les milieux sociaux considérés. Outre l'abstinence du porc qui était absolue, la coutume de la circoncision du garçonnet, opérée par un barbier, qui était générale, une observa nce du ramadân qui était assez stricte pour les deux sexes, on marquait le culte du vendredi, la première prière de l'aube au moins, le respect de la parole donnée et l'honnêteté, des manières dignes et la pratique d'une étiquette, avec des formules persanes, dès le plus jeune âge. Mais ni le vin, ni le tabac, ni l'opium n'étaient refusés. Le zakât n'était, paraît-il, pas payé fidèlement à la mosquée. Mais la mendicité était un métier socialement reconnu, au point d'être organisée en corporation, tant était forte l'obligation de charité. Remarquons que, dans les communautés de Chinois musulmans, la mendicité était réprouvée, mais la solidarité du groupe fonctionnait de manière à la rendre inutile.

Il est bien connu que l'islam du Xinjiang agricole est du sunnisme de rite hanéfite. Mais jusqu'à une époque récente, on n'avait pas assez dit combien il était - et reste - marqué par le soufisme, lequel se manifeste, entre autres, dans le culte aux mâzar, les tombes de saints. Avant la chute de Ya'kûb-beg et la laïcisation de l'autorité suprême par la Chine, le pouvoir en Kashgarie a été partiellement détenu, nous l'avons vu, par des dynasties de khôja; or ceux-ci se rattachaient par des généalogies authentiques ou apocryphes aux saints, vénérés en ces mâzar. Et, hors de la Kashgarie, le culte populaire s'était cristallisé sur des centaines de tombes de saints mythifiés, qui polarisaient un sentiment d'identité étroitement local ${ }^{46}$.

Après la transformation de la région en province chinoise, le pouvoir des responsables religieux a été abaissé, les énormes waqf appartenant aux mosquées ou aux mâzar ont été réduits, mais non pas supprimés. Les seuls spécialistes du culte qui ont gardé un statut officiel, sans plus avoir aucun rôle politique, ont été les célébrants de mosquée, les akhund; une partie d'entre eux ont rempli le rôle de kâzi - des juges islamiques choisis par les autorités chinoises dans les milieux cléricaux, de médiocres personnalités en général, pour dire le droit coranique en matière de mariage et de divorce, alors que le juge chinois tranchait en matière patrimoniale et au pénal. D'autres membres du clergé officiel étaient des môlla, qui partageaient en chaque localité le prestige social avec le beg, le fonctionnaire autochtone local grand propriétaire terrien. Ces môlla sont raillés par les fidèles pour leur avidité, leur étroitesse d'esprit et leur dureté; mais ils sont craints et obéis. Ils sont l'élément fanatique d'une société affable, joyeuse, encline à la paix.

A côté d'eux, existe une classe religieuse officieuse, la plus vénérée du peuple, celle des shaykh, dont beaucoup descendent de ces saints dont le culte se perpétue autour des mâzar et qui disposent de waqf souvent énormes. Les confréries soufies jouent un rôle social considérable : ce sont des branches de Naqshbandiyya, ainsi que Yasawiyya, 
Qâdiriyya, Uwaysîyya. Il est possible que les rivalités sanglantes des périodes troublées aient été sous-tendues par des antagonismes de confréries. De pittoresques mendiants, des qalandar et des derviches ${ }^{47}$ font partie de la vie quotidienne dans les oasis, ainsi que des sorciers diseurs de bonne aventure, des mollâ incomplets. Car la sorcellerie, appelée aussi chamanisme par ses observateurs ${ }^{48}$, fleurit pour guérir les maladies. Gengis-khan est invoqué à côté d'Allah comme démonifuge. Des vestiges de culte préislamique se remarquent d'ailleurs autour des mâzar : ainsi l'expédition Dutreuil de Rhins faisait la curieuse observation que les mâzar étaient ornés de tugh, fanions en crin de yaks ou de chevaux que portaient les soldats de Gengis-khan au XIIIe siècle et qui étaient réputés être le siège du charisme du grand-khan.

Les historiens communistes, chinois et soviétiques, ont voulu expliquer les mouvements de rébellion du Xinjiang et leurs échecs uniformément par la lutte des classes. Il est indéniable que les beg étaient prochinois pour protéger leur position, que la majorité des leaders insurgés ont été guidés par leur intérêt plus que par pure idéologie. Mais voir des "soulèvements populaires" dans les explosions périodiques de colère populaire, nées de motifs initiaux souvent accidentels, et stimulées passagèrement par des mollâ xénophobes, ne correspond guère aux faits. Car la religion justifiait le destin individuel et les inégalités sociales, celles-ci étant issues de la fortune et non d'ordres sociaux héréditaires. Le goût portait à ne travailler pas plus qu'il n'était nécessaire et à cultiver la modération. Aussi la société sédentaire du Turkestan oriental a-t-elle été le type de ces sociétés prémodernes où revenus et besoins s'équilibraient en général. Les récits sont multiples qui montrent, en temps de troubles, un peuple tiré à hue et à dia par de petites factions d'insurgés, de fanatiques ou de pillards, et qui est las de la conscription à laquelle on le contraint, des massacres de Chinois auxquels on l'incite. Les Dounganes - les Chinois de conviction musulmane, dits Hui en RPC - étaient, au Xinjiang, particulièrement habiles à enrégimenter et à faire travailler pour euxmêmes, de sorte que les sentiments de solidarité islamique n'avaient pas l'occasion de jouer entre Ouïgours et Dounganes.

Depuis la relative détente des années quatre-vingt, le culte est très vite sorti de ses cendres, tout comme en Chine propre, et les mosquées ont été réouvertes et réparées. Deux concepts, lourds de symboles, polarisent la double opposition au régime communiste et à l'occupant chinois : l'islam et l'identité ouïgoure, celle-ci étant, de nos jours, totalement acceptée selon les lignes tracées par le régime, c'est-à-dire uniforme pour tous les turcophones sédentaires de la province et directement héritée d'un empire médiéval glorieux, dont les traditions se sont perpétuées jusqu'à nos jours. Mais le réalisme vient contrebalancer les rêves d'émancipation colportés par ces concepts : alors que la présence chinoise se fait de plus en plus visible au Xinjiang, il devient évident que l'accès à la réussite sociale passe par l'apprentissage du chinois et l'acceptation des normes officielles. Les deux séductions entrent maintenant en compétition : l'identité islamique communautaire ou l'assimilation dans le milieu chinois. 


\section{BIBLIOGRAPHIE}

Références bibliographiques*

Acta Asiatica. Bulletin of the Institute of Eastern Culture, n³4, 1978 (Tôkyô) : numéro spécial consacré aux études historiques sur l'Asie centrale au Japon (en anglais).

ALLSEN, Thomas, 1983, "The Yüan Dynasty and the Uighurs of Turfan in the 13th century", in Rossabi, 1983, pp. 243-280.

BANATEANU, Tancred, 1960-61, "Notes ethnographiques sur les Ouïgours de l'oasis de Tourfan (Province du Sin-Kiang, R.P. Chinoise)", Studia et Acta Orientalia, III, Bucarest, pp. 3-14.

BAZIN, Louis, 1994, Les Turcs. Des mots, des hommes (M. Nicolas \& G. Veinstein, éd.), Paris, Editions Arguments, \& Budapest, Akadémiai Kiadó, 428 p.

BECKWITH, Christopher I., 1987 (rééd. en paperback 1993), The Tibetan Empire in Central Asia. A history of the struggle for great power among Tibetans, Turks, Arabs, and Chinese during the Early Middle Ages, Princeton Univ. Press, xxii $+281 p$.

BELLÉR-HANN, Ildikó, 1996, "Narratives and values : Source materials for the study of popular culture in Xinjiang", Inner Asia (Occasional Papers), I, n ${ }^{\circ}$, Cambridge, pp. 89-100. BELLÉR-HANN, Ildikó, 1997, "The peasant condition in Xinjiang", The Journal of Peasant Studies, XXIV, nº 4 , pp. 87-112.

Bol'shaja Sovetskaja Enciklopedija (La Grande Encyclopédie Soviétique), tome 26, 1977, article "Ujgury" (Uigurs), p. 531.

BOREI, Dorothy V., 1992, "Beyond the Great Wall : Agrocultural development in Northern Xinjiang, 1760-1820", dans J.K. Leonard \& J.R. Watt, ed., To achieve Security and Wealth. The Qing Imperial State and the Economy, 1644-1911, Ithaca (N.Y.) : Cornell Univ., pp. 21-46.

CABLE, Mildred \& FRENCH, Francesca, [1942]1984, The Gobi Desert, (1ère éd. 1942), réimpr. 1984, Londres, Virago Travellers, xxi + 303 p., illustr.

The Cambridge History of China, 1978, vol. X, Late Ch'ing, 1800-1911. Part 1, Cambridge University Press, $713 \mathrm{p}$.

The Cambridge History of China, 1980, vol. XI, Late Ch'ing, 1800-1911. Part 2, Cambridge University Press, $754 \mathrm{p}$.

CHU Wen-djang, 1966, The Moslem Rebellion in Northwest China, 1862-1878. A study of Government Minority Policy, La Haye-Paris, Mouton (reconquête du Xinjiang, pp. 163-196).

CHVYR', Ljudmila Anatol'evna, 1986, "Sravnitel'nyj ocherk tradicionnyx ukrashenij Ujgurov i sosednix narodov Central'noj i Srednej Azii" (Aperçu comparatif des parures traditionnelles des Ouïgours et des peuples voisins de l'Asie centrale et moyenne), Vostochnyj Turkestan i Srednjaja Azija $v$ sisteme kul'tur drevnego i srednevekovogo Vostoka (Le Turkestan oriental et l'Asie moyenne dans le système des cultures de l'Orient ancien et médiéval), pp. 211-250.

CHVYR', Lujdmila Anatol'evna, 1990, Ujgury Vostochnogo Turkestana i sosednie narody $v$ konce XIX-nachale XXv. (Les Oügours du Turkestan Oriental et les peuples voisins à la fin du XIXe et au début du XXe siècles), Moscou, Nauka, 301 p. (pp. 278-293; bonne bibliographie des récits de voyage et des témoignages du début de ce siècle concernant les Ouïgours). 
CHVYR', L.A., \& RESHETOV, A.M., 1992, "Sovremennye rezul'taty êtnicheskix processov v Vostochnom Turkestane" (Les résultats actuels des processus ethniques au Turkestan Oriental), dans Litvinskij, 1992, pp. 406-426.

DABBS, Jack A., 1963, History of the Discovery and Exploration of Chinese Turkestan, La Haye, Mouton \& $C^{\circ}, 255$ p. (bibliographie, pp. 185-200).

DU Shaoyuan, 1995, traduit et commenté par Marie-Lise BEFFA \& WANG Mingrong,

"Pratiques chamaniques des Ouiggours du Xinjiang", Etudes mongoles et sibériennes, XXVI, pp. 41-62.

Encyclopaedia Iranica, éditée par Ehsan Yarshater, Londres, depuis 1982, en publication jusqu'à maintenant (au tome V, 1992, article "Chinese Turkestan").

Encyclopédie de l'Islam, nouvelle édition, Leiden, depuis 1960, en publication jusqu'à maintenant.

ETHERTON, P.T., 1911, Across the Roof of the World, Londres, Constable \& $\mathrm{C}^{\circ}$, xvi $+437 \mathrm{p}$. ETHERTON, P.T., 1925, In the Heart of Asia, Londres, Constable \& $\mathrm{C}^{\circ}$, xii $+305 \mathrm{p}$.

FIORANI PIACENTINI, Valeria, 1973, "Turchizzazione ed islamizzazione dell' Asia Centrale (VI-XVI sec. dopo Cristo)", Nuova Rivista Storica (Rome), LVII, pp. 613-688.

FLETCHER, Joseph, 1968, "China and Central Asia" dans John K. Fairbank ed., The Chinese World Order, Harvard Univ. Press, pp. 206-224 et notes remarquables pp. 345-368; réimpression dans Fletcher, 1995, art. nII.

FLETCHER, Joseph, 1975, "Central Asian Sufism and Ma Ming-hsin's New Teaching", Proceedings of the Fourth East Asian Altaistic Conference, 1971, Taipei, National Taiwan Univ., pp. 75-96; réimpression dans Fletcher, 1995, art. IV.

FLETCHER, Joseph, 1978, "Ch'ing Inner Asia" et "The heyday of the Ch'ing order in Mongolia, Sinkiang and Tibet", The Cambridge History of China, X, respect. chap.2, pp. 58-90 \& chap.8, pp. 360-395, notes pp. 592-595.

FLETCHER, Joseph, 1986, "Les voies (turuq) soufies en Chine", dans A. Popovic \& G. Veinstein, dir., Les ordres mystiques dans l'islam, Paris, Editions de l'Ecole des Hautes Etudes en Sciences Sociales, pp. 13-26; cf. Fletcher, 1994.

FLETCHER, Joseph, 1994, "The Sufi Paths (turuq) in China", Etudes Orientales, XIII-XIV, pp. 55-69 (version anglaise originale de Fletcher, 1986).

FLETCHER, Joseph, 1995, Studies on Chinese and Islamic Inner Asia, Londres, Variorum (rééd. posthume sous la dir. de B.-F. Manz de dix articles de l'auteur, plus un article inédit, index).

FORBES, Andrew D.W., 1986, Warlords and Muslims in Chinese Central Asia. A Political History of Republican Sinkiang, 1911-1949, Cambridge Univ. Press, xvi + 376 p.

FORSYTH, Sir T.D., 1875, Report of a Mission to Yarkund in 1873, under command of Sir T.D.

Forsyth... with historical and geographical information regarding the Possessions of the Ameer of Yarkund, Calcutta, Foreign Dpt Press, in $-4^{\circ}, 573$ p.

GABAIN, Annemarie von, 1973, Das Leben im uigurischen Königreich von Qoco, 850-1250, Wiesbaden, O. Harrassowitz (Veröffentlichungen der Societas Uralo-Altaica, 6), 251 p. + 235 illustr.

GENG Shimin, 1981-82, "On the fusion of nationalities in the Tarim-Basin and the formation of the modern Uighur nationality", Materiala Turcica, VII-VIII, pp. 168-190 (ou Central Asian Survey, III, 1984, n4, pp. 1-4).

GLADNEY, Dru C., 1990, "The Ethnogenesis of the Uighur", Central Asian Survey, IX, n¹, pp. 1-28.

GLADNEY, Dru C., 1991, Muslim Chinese : Ethnic Nationalism in the People's Republic, Harvard Univ. Press (Harvard East Asian Monographs, 149), xxvi + 473p. (rééd. 1996). 
GLADNEY, Dru C., 1996, "Relational alterity : Constructing Dungan (Hui), Uygur, and Kazakh identities across China, Central Asia, and Turkey", History and Anthropology, IX, $\mathrm{n}^{\circ} 4$, pp. 445-477.

GOLDEN, Peter, 1992, An Introduction to the History of the Turkic Peoples. Ethnogenesis and State-formation in Medieval and Early Modern Eurasia and the Middle East, Wiesbaden, Otto Harrassowitz ("Turcologia"), 483 p.

GOLOMB, Ludwig, svd, 1959, Die Bodenkultur in Ost-Turkestan. Oassen Wirtschaft und Nomadentum, Posieux (Suisse), Anthropos-Institut (Studia Instituti Anthropos, 14), xii + 160 p. +21 pl. +5 c.

GRENARD, Fernand, 1898, Le Turkestan et le Tibet. Etude ethnographique et sociologique, formant la 2e partie de J.-L. Dutreuil de Rhins, Mission scientifique dans la Haute Asie, 1890-1895, Paris, Ernest Leroux, 476 p.

HAMADA, Masami, 1978, "Islamic Saints and their Mausoleums", Acta Asiatica, n³4, pp. 79-98.

HANEDA, Akira, 1978, "Problems of Turkicization. Problems of Islamization", Acta Asiatica, n³4, pp. 1-21.

HARMATTA, János, édit., 1994, History of Civilizations of Central Asia, vol.II, The development of sedentary and nomadic civilizations, 700 B.C. to A.D. 250, Paris, UNESCO, 573 p. HAUSSIG, Hans Wilhelm, 1992, Die Geschichte Zentralasiens und der Seidenstrasse in vorislamischer Zeit, Darmstadt, Wissenschaftliche Buchgesellschaf (1ère édit. 1983), xii + $322 \mathrm{p}$.

HOPPE, Thomas, 1995, Die ethnischen Gruppen Xinjiangs : Kulturunterschiede und interethnische Beziehungen, Hamburg, Institut für Asienkunde (Mitteilungen des Inst.f.Asienkunde Hamburg, 258), 564 p.

HORVATH, Izabella, and DU Yaxiong, 1995, "Xinjiang's population of the pre-Turkic age in light of Chinese archeology and historical sources", Eurasia Studies Yearbook, LXVII, pp. 95-108.

ISIEV, Daut Abdulumovich, 1981, Ujgurskoe gosudarstvo Jêttishar, 1864-1877 (Le royaume ouïgour de Yedîshahr, en russe), Moscou, Académie des Sciences, 91 p.

JARRING, Gunnar, 1961, "A note on Shamanism in Eastern Turkestan", Ethnos (Stockholm), pp. 1-4.

JARRING, Gunnar, 1979-80, Matters of ethnological interest in Swedish Missionary Reports from Southern Sinkiang, formant Scripta Minora, Lund (Suède), 1979-1980, n4, 21 p. JARRING, Gunnar, 1985-86, Dervish and Qalandar. Texts from Kashgar, formant Scripta Minora, 1985-1986, n², 39 p.

JARRING, Gunnar, 1991, "The Uighurs of Xinjiang : Their place in Swedish history and research", Journal. Institute of Muslim Minority Institute, XII, n¹, pp. 105-113.

JARRING, Gunnar, 1991-92, Garments from top to toe. Eastern Turki texts relating to articles of clothing, formant Scripta Minora, 1991-1992, n², 93 p.

JOYCE, T.A., 1912, "Notes on the physical anthropology of Chinese Turkestan and the Pamirs", Journal of the Royal Anthropological Institute, XLII, pp. 450-484 (ou en introduction à Sir Aurel Stein, Serindia, 1921).

KIM Ho-dong, 1993, "The cult of Saints in Eastern Turkestan : The case of Alp Ata in Turfan", Proceeding of the 35th PIAC, 1992, Taipei, pp. 199-226.

KRIPPES, Karl, 1991, "Sociolinguistic notes on the turcification of the Sogdians", Central Asiatic Journal, XXXV, n²1-2, pp. 67-80. 
KUROPATKIN, A.N., 1882, Kashgaria. Historical and Geographical sketch of the country; its military strength, industries and trade, Calcutta, Thacker, Spink and $\mathrm{C}^{\circ}$, in $-4^{\circ}$, viii $+255 \mathrm{pp}$. (original russe de 1877).

LATTIMORE, Owen, 1950, Pivot of Asia. The Inner Asian frontiers of China and Russia, Boston, Little, Brown and $\mathrm{C}^{\circ}$, xiv $+288 \mathrm{p}$.

LITVINSKIJ, Boris Anatol'evich, éd., 1992, Vostochnyj Turkestan v drevnosti i rannem srednevekov'e : Etnos, jazyki, religii (Le Turkestan Oriental dans l'antiquité et au début du moyen-âge; ethnies, langues, religions), Moscou, Nauka, 687 p.

LE COQ, Albert von, (1928), 1982, Von Land und Leuten in Ostturkestan. Berichte und Abenteuer der 4. deutschen Turfanexpedition, Leipzig, Verlag der J.C. Hinrichs'schen, 3e éd. 1928 , réimprimé 1982 , viii +183 p.

LIU Kwang-ching \& SMITH, Richard J., 1980, "The military challenge : the north-west and the coast", The Cambridge History of China, XI, chap.4, pp. 202-273 et notes bibliographiques, pp. 610-612.

MAILLARD, Monique, 1973, Essai sur la vie matérielle dans l'oasis de Tourfan pendant le haut Moyen Age, formant numéro spécial des Arts Asiatiques, XXIX, 187 p. + 237 illustr. + Xvi planches.

MALOV, Sergej Efimovich, 1967, Jazyk Zholtyx Ujgurov (La Langue des Ouïgours Jaunes), Moscou, Nauka, 218 P.

MENGES, Karl H., 1995, "On recent anthropological excavations in Eastern Türkistan", Central Asiatic Journal, XXXIX, n¹, pp. 97-101.

Narody Srednej Azii i Kazaxstana (Les peuples de l'Asie Moyenne et du Kazakhstan), tome II, 1963, Moscou.

Narody Vostochnoj Azii (Les peuples de l'Asie orientale), 1965, Moscou-Léningrad, Nauka ("Science", Maison d'édition de l'Acad. des Sciences d'URSS), dans la collection Narody Mira : Etnograficheskie Ocherki (Les peuples du monde : Aperçus ethnographiques).

PELLIOT, Paul, 1912, "Les influences iraniennes en Asie centrale et en Extrême-Orient. Leçon d'ouverture du Cours de langues, histoire et archéologie de l'Asie centrale, 4 décembre 1911", Revue d'Histoire et de Littérature religieuses, III, n², pp. 97-119. PELLIOT, Paul, ca. 1930, La Haute Asie, Paris, Expédition Citroën Centre-Asie, 37p. PRITSAK, Omeljan, 1959, "Das Neuuïgurische", Philologiae Turcicae Fondamenta, tome I, Wiesbaden, Franz Steiner, pp. 525-563.

RACHEWILTZ, Igor de, 1983, "Turks in China under the Mongols : A preliminary investigation of Turco-Mongol relations in the 13th and 14th centuries", dans Rossabi, 1983, pp. 281-310.

ROSSABI, Morris, 1975, China and Inner Asia. From 1368 to the Present Day, Londres, Thames \& Hudson, 320 p.

ROSSABI, Morris, éd. 1983, China among Equals. The Middle Kingdom and its Neighbors, 10th-14th Centuries, Berkeley, etc., Univ. of California Press, xiv + 419 p.

RUDELSON, Justin Jon, 1991, "Uighur historiography and Uighur ethnic nationalism", dans Ingvar Svanberg, ed., Ethnicity, Minorities and Cultural Encounters, Uppsala, Centre for Multiethnic Research, Uppsala Univ. (Uppsala Multiethnic Papers, 25), pp. 63-82. SAGUCHI Tôru, 1978, "Kashgaria", Acta Asiatica, n³4, pp. 61-78 (et pp. 102-104, bibliographie des travaux de Saguchi, dont beaucoup portent sur le Turkestan oriental et sur les Ouïgours).

SAGUCHI Tôru, 1986, "Historical development of the Sarigh Uyghurs", Memoirs of the Tôyô bunko (Tôkyô) LXIV, pp. 1-26. 
SAMOLIN, William, 1964, East Turkistan to the twelfth century. A brief political survey, La Haye, Mouton \& $\mathrm{C}^{\circ}, 100 \mathrm{p}$.

SCHWARZ, Henry G., 1976, "The Khwâjas of Eastern Turkestan", Central Asiatic Journal, XX, nº 4 , pp. 266-296.

SHAW, Robert, [1871], 1984, Visits to High Tartary, Yarkand and Kashgar, 1ère éd., Londres, John Murray, 1871, réédité avec introd. par Peter Hopkirk, Hong Kong, Oxford Univ. Press, $x v i+486$ p. + xv pl. et 2 c. h.t.

SHINMEN Yasushi, 1993, "Research in Japan on Islamic Central Asian History", Asian Research and Trends, III, pp. 43-65.

SINOR, Denis, édit., 1990, The Cambridge History of Early Inner Asia, Cambridge Univ. Press, $\mathrm{x}+518 \mathrm{p}$.

SINOR, Denis, 1985, "Some components of the civilization of the Turks (6th to 8 th century A.D.)", Altaistic Studies, Uppsala, pp. 145-159.

STEIN, Sir Aurel, 1921, Serindia. Detailed Report of Expeditions in Central Asia and Westermost Asia, 5 vol. (Vol. V : 94 cartes du Xinjiang, d'après les relevés et explorations de Sir Aurel Stein en 1906-1908).

THORAVAL, Joël, 1990, "Le concept chinois de nation est-il obscur? A propos du débat sur la notion de "minzu" dans les années 1980", Bulletin de Sinologie, n65, mars, pp. 24-39.

TOGAN, Isenbike, 1992, "Islam in a changing society. The Khojas of Eastern Turkestan", Jo-Ann Gross, ed., Muslims in Central Asia, Durham, Duke Univ. Press, xiv + 224 p. VOSKRESSENKI, Alexei D., 1994-a, "Current concepts of Sino-Russian relations and frontier problems in Russia and China", Central Asian Survey, XIII, n³, pp. 361-381. VOSKRESSENKI, Alexei D., 1994-b, "Recent Chinese interpretations of Tsarist Russia's policy in Central Asia and Far East", Central Asia Monitor, 1994, n6, pp. 22-30.

WARIKOO, K.B., 1985, "Chinese Turkestan during the nineteenth century. A socioeconomic study", Central Asian Survey, IV, n³, pp. 75-114.

WEI Liangtao, 1993, "An illusion in the history of the Western Region. The 'Holy State of Islam' or the 'Khoja period'", Social Sciences in China, XIV, n³, pp. 126-140 (traduction de "Xiyushishang-di yige huanying", dans Zhongguo shehui kexue, 1992, n4, pp. 101-119). Weiwuerzu jianshi (Brève histoire du peuple oü̈gour), 1991, ouvrage collectif, Ürümchi, Xinjiang renmin chubanshe, 409 p. + illustr.

Weiwuerzu shiliao jianbian (Dossier succinct de documents historiques sur le peuple ouïgour), (1958) 2 réédit. 1981, par Feng Jiasheng, Cheng Suluo et Mu Guangwen, Pékin, Minzu chubanshe, 475 p. en 2 vol.

YANG Shengmin, 1991, Huiheshi (Histoire ouïgoure), Changchun, Jirin jiaoyu chubanshe ("Zhongguo shaoshu minzu wenku", Collection Minorités chinoises), 226p. + illustr. ZARCONE, Thierry, 1996, "Le Turkestan chinois", dans A. Popovic et G. Veinstein, dir., Les Voies d'Allah. Les ordres mystiques dans le monde musulman des origines à aujourd'hui, Paris, Fayard, pp. 268-273.

ZAXAROVA, I.V., 1959, "Material'naja kul'tura Ujgurov Sovetskogo Sojuz" (La culture matérielle des Uigurs de l'Union soviétique), Sredneaziatskij êtnograficheskij sbornik (Recueil ethnographique de l'Asie Moyenne), tome II, Moscou, Académie des Sciences de l'URSS, pp. 215-298 (de la même, un article dans Narody Vostochnoj Azii, 1965). ZAXAROVA, I.V., 1963, "Ujgury" (Les Ouïgours), Narody Srednej Azii i Kazaxstana, II, pp. 488-526.

ZAXAROVA, I.V. \& TIXONOV, D.I., 1965, "Ujgury", Narody Vostochnoj Azii, pp. 616-630. 
ZOTOV, O.V., 1991, "Gosudarstva Vostochnogo Turkestana i genezis Sin'czjana" (Les Etats du Turkestan Oriental et la génèse du Xinjiang), Vostok (Orient), nº, pp. 22-32.

\section{NOTES}

1. Sur la théorie des nationalités et ses implications dans la Chine des années quatrevingt : Thoraval, 1990; Gladney, 1991, notamment pp. 76-96, 300-321.

2. Ainsi l'histoire des Ouïgours médiévaux, Huiheshi de Yang Shengmin, 1991, a été publiée dans une "Collection des minorités chinoises". Toutes les publications de la RPC sur le sujet vont dans le même sens, par exemple Weiwuerzu shiliao jianbian (Dossier succinct de documents historiques sur le peuple ouïgour), paru pour la première fois en 1958 et réimprimé deux fois coup sur coup en 1981, ou Weiwuerzu jianshi (Brève histoire du peuple ouïgour), 1991.

3. Le climat excessif et les obstacles du relief montagneux et des déserts ont été abondamment dépeints, dans leur horreur, par tous les voyageurs qui ont pénétré dans la région avant l'ère des avions, des routes goudronnées et des trains. Rappel des nombreuses expéditions de reconnaissance et d'exploration occidentales dans le Turkestan oriental depuis le XVIIIe siècle dans Dabbs, 1963 (malheureusement les récits russes, qui occupent une part importante du domaine, y sont cités principalement dans des traductions anglaises, s'il en existe). Description géographique technique :

Encyclopaedia Iranica, V, 1992, pp. 461-463; description efficace par un témoin hors ligne, Pelliot, ca. 1930, pp. 1-2. Présentation de géographie humaine et d'anthropologie physique : Grenard, 1898. Quant à la bibliographie concernant l'histoire de l'Asie centrale aux époques contemporaines de l'Antiquité et du Moyen Age de l'Occident, elle est considérable, particulièrement bien fournie en travaux linguistiques, philologiques, archéologiques; on en trouvera un beau choix dans Sinor, 1990, pp. 424-494, et dans Haussig, 1992, pp. 262-282, mais arrêté, dans l'un et l'autre cas, à la fin des années 1970. Pour l'époque 700 av. JC à 250 ap. JC : voir Harmatta, 1994; pour celle allant du début de notre ère au XIIe siècle : Samolin, 1964. Sur les conclusions tirées par les archéologues chinois de leurs découvertes : Horvath \& Du, 1995.

4. Sur ces momies, en un état de conservation exceptionnel, et le programme sinoaméricain d'analyse de leur appartenance génétique, physique et ethnologique mis en train en 1991 : rapport de l'initiateur de l'entreprise et un de ses principaux acteurs, Victor Mair (de l'Université de Pennsylvanie), dans Inner Asia Report (Indiana Univ.), n¹3, printemps 1994, pp. 10-13; rappel des différentes trouvailles : Mengès, 1995.

Photos et description des momies dans Discover, avril 1994; photos et présentation de la "Beauté de Loulan", datant de ca 2000 avant notre ère, dans National Geographic, 189, n³, mars 1996.

5. Histoire partielle de ces Indo-Européens, des Yuezhi surtout, dans Haussig, 1992, pp. 100-138, et Sinor, 1990, pp. 151-176 (cette dernière contribution, par A.K. Narain, est corrigée et complétée par Jan Nattier, Journal of the American Oriental Society, CXI, $\mathrm{n}^{\circ} 4$, 1991, pp. 776-784, notamment pp. 778-779). Sur la turquisation du sogdien :

Krippes, 1991. Voir aussi la belle contribution de V. Mair \& P.O. Skaervo, Encyclopaedia Iranica, V, 1992, pp. 463-469.

6. Sinor, 1990, pp. 118-149 (article par Yü Ying-shih, qui y traite surtout d'histoire événementielle et d'un point de vue chinois, sans toucher à la délicate question de 
l'ethnogenèse et de l'appartenance linguistique des dirigeants Xiongnu); Haussig, 1992, pp. 139-160, toujours attentif aux témoignages philologiques et culturels.

7. Sinor, 1990, pp. 291-297; Haussig, 1992, pp. 163-164.

8. Sinor, 1990, pp. 285-316 (par l'éditeur, D. Sinor); Haussig, 1992, pp. 161-182.

9. Sinor, 1990, pp. 317-342 (par Colin Mackerras, qui limite son propos au khanat ouïgour de Mongolie).

10. La civilisation ouïgoure de Qocho est décrite, avec une abondante iconographie, par Gabain, 1973, et Maillard, 1973.

11. Cf. H.A.R. Gibb, The Arab Conquest in Central Asia, Londres, The Royal Asiatic Society, 1923.

12. Sur les Karakhanides : Sinor, 1990, pp. 342-370 (beau chapitre par Peter B. Golden); également Golden, 1992. Sur la turquisation et l'islamisation : Haneda, 1978; Fiorani Piacentini, 1973 (couvrant l'ensemble de l'Asie centrale). Très bon exposé des conditions pratiques de l'islamisation en Ouigouristan : Kim Ho-dong, 1993.

13. Cf. Encyclopédie de l'Islam, IV (1976); pp. 727-729. Sur la date du Dîwân : Bazin, 1994, pp. 384-387 - une première version date de 1972, l'ultime (et quatrième) version remonte à 1083, et l'unique copie connue est de deux siècles postérieure, de 1266 .

14. Enc. de l'Islam, V (1982), pp. 542-543.

15. Allsen, 19873; Rachewiltz, 1983.

16. D'Erd' (= Györgi) Kara, Knigi mongol'skix kochevnikov (Les livres des nomades mongols, en russe), Moscou, Acad. des Sciences d'URSS, 1972, p. 19.

17. Allsen, 1983, pp. 258-260; Encyclopaedia Iranica, V (1992), pp. 172-173.

18. Geng Shimin, 1982.

19. Description d'après les récits des voyageurs et les sources chinoises des SarighYugur : Saguchi, 1986. Sur leur langue et leur littérature orale : Malov, 1967. Grâce à Dru Gladney, je viens d'apprendre qu'une étudiante, Sabrina Stahlberg, qui a fait un travail de terrain sérieux chez les Yugurs du Gansu, en est arrivé à la conclusion qu'ils ne sont pas les descendants directs des Ouïgours médiévaux, mais qu'ils représentent un groupe hybride, né après la chute du royaume Xi-Xia au XIIIe siècle, et qu'ils ont combiné des influences tibétaines, turques, chinoises.

20. Bon récit de l'histoire des khôja : Schwarz, 1976; plus schématiquement : Mme Togan, 1992 (qui, remarquons-le, paraît ignorer Schwarz, 1976); également Encyclopaedia Iranica, V, 1992, pp. 474-476. Une vision basée sur une connaissance fine des sources disponibles et sur une évaluation des qualités et des défauts desdites sources, ainsi que sur une critique acerbe des positions soutenues par les historiens occidentaux : Wei Liangtao, 1993.

21. Sur la conquête du Turkestan oriental par les Qing au XVIIIe siècle :Fletcher, 1968, pp. 218-221; Rossabli, 1975, pp. 148-149.

22. Cf. Borei, 1992.

23. Cf. Fletcher, 1968, pp. 221-223, et surtout 1978, pp. $87-90$ et 360-395, sans oublier les notes pp. 594-595.

24. Sur ce titre : Encyclopédie de l'Islam, I, 1960 ou années suivantes, p. 458.

25. Sur le titre militaire d'atalik : Encyclopédie de l'Islam, I, p. 751 sous article ata, synonyme d'atabeg, op. cit., pp. 753-754. Ghâzi : op. cit., II, 1965, pp. 1068-69.

26. Une étude interne du gouvernement de Ya'kûb-beg : Isiev, 1981; et surtout une thèse de doctorat très vantée, mais qui, autant que je sache, n'a malheureusement pas été publiée : Kim Ho-dong, The Muslim Rebellion of the Kashgar Emirate in Chinese Central Asia, 1864-1877, PhD Dissertation, Harvard Univ., 1986 (l'auteur, coréen, s'avère 
être un des meilleurs spécialistes du Turkestan oriental au XIXe siècle : cf. Kim HoDong, 1993). Une vision chinoise de la "révolte" de Ya'kûb-beg et de son écrasement : Chu-Wen-djang, 1966. La reconquête chinoise par les Qing : Liu Kwan-ching \& Smith, 1980.

27. La spécificité de la culture politique propre à chacune des sous-régions du Turkestan Oriental au XXe siècle est bien mise en lumière par Rudelson, 1991.

28. Cf. Encyclopédie de l'Islam, I, ca. 1960, p. 435 (notice par W. Barthold, reprise de la première édition de l'Encyclopédie).

29. Cf. Encyclopédie de l'Islam, IX, 1995, pp. 69-71, long article sârt originellement de W. Barthold dans la première édition, complété et élargi par Mme M.E. Subtelny.

30. La montée des divers nationalismes turkestanais à l'époque de la République est dépeinte par Forbes, 1986.

31. Bol'shaja Soveckaja Ênciklopedija, tome XXVI, 1977, p. 531, colonne 1579.

32. Op. cit., p. 530, col. 1578.

33. Forsyth, 1875, p. 95 (la Grande Encyclopédie Soviétique, elle, stipule expressément que "le ouïgour [sous-entendu moderne] n'apparaît pas génétiquement être la continuation de l'ancien ouïgour"). Remarquons que Robert Shaw, qui avait fait un premier voyage d'exploration en Kashgarie en 1868 et qui, en 1869, participait à la première mission Forsyth, dénomme les autochtones des "Musulmans", sans plus, dans ses mémoires datant de 1871 (Visits to High Tartary), suivant en cela l'usage local.

34. Sur l'histoire de cette époque : Forbes, 1986.

35. Sur la construction de l'identité ethnique ouïgoure dans le monde contemporain : Gladney, 1990 et 1996; d'un point de vue ethnologique : Hoppe, 1995. Le récit d'un témoin de l'époque républicaine très fiable : Lattimore, 1950.

36. On trouve un exemple de l'argumentation chinoise selon laquelle "nos minorités anciennes ont régné sur toute l'Asie" dans la revue d'études historiques, Lishi yanjiu, 1977, n³, pp. 126-141 (à propos des Xiongnu).

37. Communiqué de l'Agence Xinhua du 18 février 1980 (dans SWB/FE/6362, B/II/6 du 5 mars 1980).

38. Bon exposé des mélanges ethniques qui se sont opérés au cours de deux millénaires au Turkestan Oriental : Geng Shimin, 1981-82; cf. Hoppe, 1995, tableau, p. 66, et pp. 69-80, liste et description des multiples peuples, d'origine diverse, qui ont été assimilés abusivement aux Ouïgours par la théorie officielle des minorités en RPC. Sur les composantes ethniques, le substrat indo-européen notamment, que détectent les enquêtes d'anthropologie physique : Joyce, 1912.

39. Présentation linguistique du "nouvel ouïgour", par exemple, par Pritsak, 1959.

40. Un classique sur les influences indo-européennes présentes dans le Turkestan préislamique est Pelliot, 1912. Sur les composantes non turques (certaines sans doute samoyèdes) dans la civilisation des Turcs des VIe-VIIIe siècles : Sinor, 1985.

41. Sur l'histoire de la présence tibétaine dans l'Asie centrale médiévale, voir le travail riche et original de Beckwith, 1987/1993.

42. Liste commentée : Dabbs, 1963.

43. Bellér-Hann, 1997, et infra, son article dans ce même dossier. Mme Bellér-Hann (1996) a eu le louable propos de soumettre à une critique impartiale les documents disponibles sur la vie populaire au Xinjiang et d'y débusquer les préjugés anti-indigènes qui y affleurent. Infortunément, sa bibliographie passe sous silence les titres essentiels en français (Grenard, 1898 et Banateanu,1960-61), peut-être parce que ceux-ci lui paraissent exempts de vice, mais il aurait fallu le préciser. 
44. Soulèvements décrits par Forbes, 1986.

45. D'après J. Thomson Golver, "Present-day Kashgaria", Journal of the Royal Central Asian Society (Londres), XXIV, n ${ }^{\circ}$, juillet 1937, p. 439.

46. Kim Ho-dong, 1993, prenant pour exemple le culte rendu, depuis le XVe siècle à Alp Ata dans l'oasis de Turfan, a attiré l'attention sur l'importance des mâzar locaux dans la persistance du culte populaire.

47. Description autochtone des derviches et des qalandar : Jarring, 1985-86.

48. Du Shaoyuan, propagandiste chinois au Xinjiang dès les années cinquante, décrit une séance chamaniste (Du Shaoyuan, 1995); et il est convaincu que le rite auquel il a assisté constitue le reliquat du chamanisme médiéval des anciens Ouïgours. Autres mentions du chamanisme au Xinjiang et bibliographie des sources à ce sujet : Jarring, 1961.

* Les bibliographies incluses dans les ouvrages ici mentionnés permettront de compléter le choix de titres, très succinct, qui a été opéré pour la présente contribution. Par souci d'allègement et de simplification, aucun titre en japonais n'a été cité, malgré l'importance de plusieurs d'entre eux (mention des principaux travaux japonais afférents au sujet dans Acta Asiatica, n³4 et dans Shinmen, 1993), et peu de titre en chinois (les principaux sont relevés dans les publications de D. Gladney). 